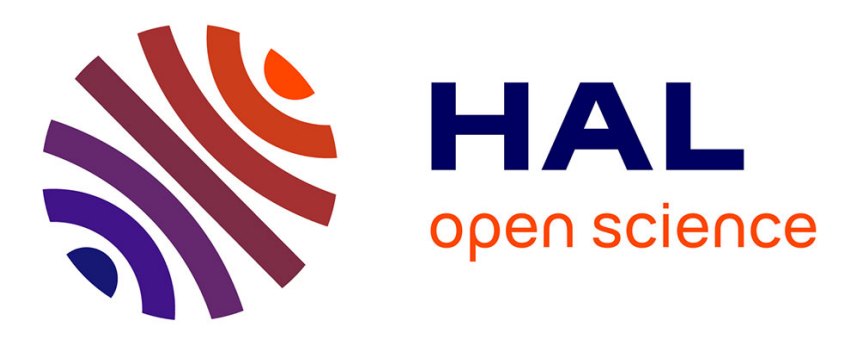

\title{
Micromechanical contributions to the behaviour of cement-based materials: two-scale modelling of cement paste and concrete in tension at high temperatures
}

Frédéric Grondin, Hélène Dumontet, Abdelwahid Ben Hamida, Hocine Boussa

\section{- To cite this version:}

Frédéric Grondin, Hélène Dumontet, Abdelwahid Ben Hamida, Hocine Boussa. Micromechanical contributions to the behaviour of cement-based materials: two-scale modelling of cement paste and concrete in tension at high temperatures. Cement and Concrete Composites, 2011, 33, pp.424-435. 10.1016/j.cemconcomp.2010.11.004 . hal-01007038

\section{HAL Id: hal-01007038 \\ https://hal.science/hal-01007038}

Submitted on 17 Feb 2018

HAL is a multi-disciplinary open access archive for the deposit and dissemination of scientific research documents, whether they are published or not. The documents may come from teaching and research institutions in France or abroad, or from public or private research centers.
L'archive ouverte pluridisciplinaire HAL, est destinée au dépôt et à la diffusion de documents scientifiques de niveau recherche, publiés ou non, émanant des établissements d'enseignement et de recherche français ou étrangers, des laboratoires publics ou privés. 


\title{
Micromechanical contributions to the behaviour of cement-based materials: Two-scale modelling of cement paste and concrete in tension at high temperatures
}

\author{
F. Grondin ${ }^{\mathrm{a}, *}, \mathrm{H}$. Dumontet ${ }^{\mathrm{b}}$, A. Ben Hamida ${ }^{\mathrm{b}}, \mathrm{H}$. Boussa ${ }^{\mathrm{c}}$ \\ anstitut de Recherche en Génie Civil et Mécanique (GeM), UMR-CNRS 6183, Ecole Centrale de Nantes, Nantes, France \\ ${ }^{\mathrm{b}}$ Institut Jean le Rond d'Alembert, UMR-CNRS 7190, Université Pierre et Marie Curie, 4 Place Jussieu 75252 Paris Cedex 5, France \\ ${ }^{\mathrm{c}}$ Division MOD-EVE, Centre Scientifique et Technique du Bâtiment, 84 Avenue Jean Jaurès, Champs-sur-Marne, 77447 Marne-la-Vallée Cedex 2, France
}

\begin{abstract}
A numerical multiscale model is proposed to study the thermo-hydro-mechanical behaviour of cement-based materials. This model is based on a finite element approach coupled to a numerical generation of the material microstructure. A homogenization method is used to calculate the macroscopic thermo-hydromechanical properties and the macroscopic hydraulic properties of heterogeneous materials according to the components properties.

At the microscopic scale, numerical simulations have been carried out on cement pastes and the hydraulic flows, strain and stress fields are localized. A recent study has been carried out on damage of concrete structures under fire conditions in compression. In this paper, the micromechanical approach is applied for the calculation of the thermo-mechanical properties of concrete under a tensile load at high temperatures and to localize damage within the microstructure. The equivalent permeability is com-puted in order to estimate the increase in the leakage rate, which is an important parameter for the risk analysis of nuclear power plants. We note a strong increase in permeability in the damaged cement paste of concrete.
\end{abstract}

Keywords: Micromechanics, Thermo-hydro-mechanical behaviour Cement-based materials, High temperature, Permeability, Damage, Tension

\section{Introduction}

The service-life of concrete structures depends on the behaviour of concrete in their environment and on the concrete mixture. Indeed, the choice of the cement type, the aggregate type or the water-to-cement $(w / c)$ ratio influence concrete behaviour. To prevent an accident in concrete structures (for instance in reactors cells of nuclear power plants), civil engineers have to build structures which can resist thermal increases and mechanical load due to the formation of a high volumetric pressures at high temperatures. This study is devoted to the behaviour of cementbased materials in tension within the range $20-600^{\circ} \mathrm{C}$ of high temperature.

In order to predict the degradation of concrete under fire conditions, many studies have been performed to identify the presumed causes [1-4]. Experimental tests have confirmed concrete brittleness at high temperatures, particularly under a mechanical load $[5,6]$. Experimental observations have allowed to localize damage in the concrete microstructure and to assess the diverse origins in damage: mechanical, thermal and chemical $[7,8]$. From $100^{\circ} \mathrm{C}$, the capillary pore water changes into water vapor and the cement

\footnotetext{
* Corresponding author.

E-mail address: frederic.grondin@ec-nantes.fr (F. Grondin).
}

hydrates begin to dessicate. Then, from $200^{\circ} \mathrm{C}$ the water vapor fills out the concrete which leads to a shrinkage phenomenon of the cement paste. At higher temperature values, the dehydration of cement hydrates takes place which leads to the nucleation of cracks in the cement paste. Also, aggregates are sensitive to temperature increases. According to their physical properties they can swell with temperature (limestone aggregates) or shrink (silicious aggregates). The difference in the behaviour of aggregates and the cement paste contributes to the cracking process of the material at high temperatures. Several experimental studies have shown that the deformation of concrete during heating is strongly influenced by the simultaneous presence of an external mechanical (compressive) loading. An additional strain component denoted "Transient Thermal Creep" (TTC) has been measured. Several hypotheses have been advanced to explain the mechanism behind the TTC. Parrot [9] considers that this phenomenon is due to an accelerated polymerization in the Calcium Silicate Hydrates (C-S-H) by the effect of compression and increase of temperature. Anderberg and Thelandersson [10] introduce the concept of the thermo-mechanical interaction. Khoury et al. [3] attribute the TTC to a relaxation and a redistribution of the thermal stresses. Sabeur and Meftah [11] consider that the combined effect of creep and drying creep of dehydration is the basis for the development of the TTC. Bazant et al. [12] explain the transient thermal creep by 
two mechanisms, the first operating at the macroscopic scale due to microcracking and thermal damage; the second mechanism is due to incompatibilities at the nanopores scale (in $\mathrm{C}-\mathrm{S}-\mathrm{H}$ ). Gawin et al. [13] connect the TTC to the thermo-chemical damage of the cement paste and to the induced micro-cracks during heating. Studies performed at the mesoscale by Grondin et al. [14,15] and Menou et al. $[16,17]$ have shown that this phenomenon is mainly attributed to the aggregates-paste incompatibility.

However, most of these studies have concerned the behaviour of concrete at high temperatures under a compressive load and there has been little analysis on its tensile behaviour under the same thermal conditions [18-20]. Experimental tests for concrete in tension are not common. So, the test devices are not adapted for the characterization of the failure properties of concrete in tension. The few results given in the literature [18-20] show a deformation evolution vs. the load. In these tests, when the critical stress is reached the concrete shows a fragile behaviour. Therefore, no experimental tests have been suggested to measure the permeability of concrete under these conditions.

Numerical approaches seem to be the best way to characterize the failure properties of concrete in tension at high temperatures. Some numerical models have been adapted to predict the thermo-hygro-mechanical behaviour of concrete under different conditions from the pioneering works of Coussy [21] and Schrefler [22]. Some of these models have been used to calculate the strain and damage of concrete at high temperatures under a compressive load [13,23-26] and its permeability [27]. Other models have been performed considering thermal, chemical and mechanical degradation $[28,29]$ or chemo-plasticity [30]. But, these models are based on a macroscopic representation of concrete and on a semi-empirical behaviour law. In this context some parameters are calibrated on experimental tests and it is not possible to show the influence of the microstructure on the damage and the hydraulic flow evolutions. In recent works, multiscale approaches coupled with homogenization methods have been applied to studying the behaviour of concrete and granular material; see for example [15,31-33]. These approaches give the possibility to access to local strain at the microstructure scale. Coupled to damage models, they enable simulation of the progressive deterioration of the microstructure at different temperature levels [34,15].

Some numerical methods have been proposed to study specific behaviour of cement-based materials [35-39]. But these models are not performed to couple the calculation of permeability with a damaged thermomechanical behaviour law. In this study, a close method presented in [15] is used. The model (Digital Concrete Model [40]) is based on a computation, by the finite element method, of the microstructure of the material. All heterogeneities (aggregates, sand grains or pores) are computed in a Representative Elementary Volume (REV) and are distributed by a random process according to their real size distribution. One of specificities is the simplicity of the finite element mesh reduced to a regular grid. So, the model can be either applied at different scales: mesoscopic (concrete) and microscopic (cement paste). From this mesh, various behaviour laws can be chosen for the different phases, in particular thermo-hydro-mechanical laws with damage have been implemented. We consider inclusions distributed in a matrix with different properties according to the scale of the study. The approach is based on the knowledge of the microstructure properties. On the cement paste scale, the complex microstructure is formed by different phases which are the porosity, the residual unhydrated phases (cement clinker) and hydrated phases created during the chemical reaction between clinker and water. Different microstructure of cement pastes at the end of the hydration process have been computed. Homogenization calculations have been made to assess the elastic properties and the hydro-mechanical properties which are difficult to determine experimentally; for instance, the
Biot's coefficient [41]. Also, the permeability coefficient, which depends highly on the injected fluid, has been calculated. On the concrete scale, a coupling of the model with a damage model [42] has been made to study the failure properties of concrete. The damage and the effective permeability of High Strength Concrete (HSC) at high temperatures in tension have been calculated.

First of all, the Digital Concrete model and the solved mechanical and hydraulic problems are described. Secondly, analyses at the cement paste scale are conducted to demonstrate the capabilities of the model to give effective properties to concrete phases on an upper scale. A description of the cement paste microstructure with hydraulic and geometrical properties of each of the phases is given in detail. In the next section, an application to concrete in tension subjected to high temperatures is presented and the results are compared to experimental measurements: total strain, homogenized mechanical properties and the effective permeability. Finally, local analyses of the damage variable and of the fluid flow are presented.

\section{The digital concrete model}

\subsection{The representative elementary volume}

The Digital Concrete Model has been developed with the objective to have a 'realistic' representation of cement-based materials by taking into account the random size distribution of heterogeneities. So, in previous applications on the behaviour of concrete, the concrete microstructure is represented as a multi-phase material with successions of three material phases (cement paste, pores, aggregate of various sizes) spatially distributed in a random way. Each phase is characterized by a set of physical and geometrical parameters such as: the volume fraction, the unit size (aggregate or pore diameter), and physico-mechanical properties. A specific algorithm has been developed to make a spatial and random distribution of these phases on the basis of a F.E. grid. Details are given in [15].

The REV dimensions and the finite element size are chosen in accordance with the material characteristics and the problem type. Generally the REV dimensions are taken equal to four times the largest inclusion diameter and the finite element size is equal to 0.8 times the smallest inclusion diameter. Calculations have been performed to check the stability of results with these dimensions for different inclusion distributions [15].

\subsection{The non-linear thermo-hydro-mechanical behaviour}

The REV $V$ of a heterogeneous material is formed by two media: a matrix defined by the medium $V_{m}$ and inclusions defined by the medium $V_{r}$. With the objective to model the behaviour of damaged cement-based materials, phases have a damage elastic behaviour. An uniform temperature $\Delta T$ and an uniform strain field tensor $\underline{E}$ are applied on the surface of $V$. These loads imply local displacements fields $\underline{u}(y)$, local strain fields $\underline{\varepsilon}(\underline{y})$ and local stress fields $\underline{\sigma}(y)$ in each point $y$ of $V$ which are linked by the following behaviour law:

$\underline{\underline{\sigma}}(\underline{y})=\underset{\sim}{C}(\underline{y}, \underline{\underline{\varepsilon}}(\underline{y})):(\underline{\underline{\varepsilon}}(\underline{y})-\underline{\underline{\alpha}}(\underline{y}, T) \Delta T)-p_{c} \underline{\underline{\delta}}$

where $C(y, \underline{\varepsilon}(y))$ is the stiffness tensor of the material phases depending on the strain of phases and $\underline{\alpha}(y, T)$ the thermal expansion tensor of the material phases depending on the temperature. $p_{c}$ is the capillary pressure which is equal to 0 in a solid medium. The stiffness tensor is depending on the strain state as follow [15]:

$\underset{\sim}{C}(\underline{y}, \underline{\underline{\varepsilon}}(\underline{y}))=\left[1-\eta d^{s}(\underline{\underline{\varepsilon}}(\underline{y}))-(1-\eta) d^{d}(\underline{\underline{\varepsilon}}(\underline{y}))\right] \underset{\sim}{C}(\underline{y})$ 
where $C(y)$ is the initial stiffness tensor of the material phases and $d^{s}$ and $\tilde{d}^{d}$ the spherical and the deviatoric damage variables which correspond respectively to the local slip in micro-cracks and the hydrostatic state. $d^{s}$ and $d^{d}$ vary between 0 (undamaged material) and 1 (total damage). The variable $\eta$ is calibrated according to the material and to the test. According to the Mazars's model [43], Ung Quoc and Mounajed [42] have developed the damage model MODEV and have suggested these following evolutions of the spherical and the deviatoric damage variables:

$d^{s}=1-\frac{\tilde{\varepsilon}_{0}^{s}}{\tilde{\varepsilon}^{s}} \exp \left[-B_{t}\left(\tilde{\varepsilon}^{s}-\tilde{\varepsilon}_{0}^{s}\right)\right]$

$d^{d}=1-\exp \left[-B_{c}\left(\tilde{\varepsilon}^{d}-\tilde{\varepsilon}_{0}^{d}\right)\right]$

where $B_{t}$ is a damage parameter related to the fracture energy to limit the mesh sensitivity, $B_{c}$ a material parameter associated to the compressive strength and $\tilde{\varepsilon}^{s}$ and $\tilde{\varepsilon}^{d}$ are respectively the spherical and the deviatoric equivalent strains given in [42] and in [15].

In this work, the effective moduli method [44] is retained and the macroscopic strain $\underline{E}$ is applied through a given displacement $\underline{u}$ on the boundary $\partial V$ of the REV:

$\underline{u}=\underline{\underline{E}} \cdot \underline{y} \quad$ on $\quad \partial V$

The non-linear homogenized behaviour law is defined by the relation between the average stress fields $\underline{\underline{\Sigma}}=\langle\underline{\underline{\sigma}}\rangle_{V}$ and the average strain fields $\langle\underline{\varepsilon}\rangle_{V}$ where $\underline{\underline{\sigma}}$ and $\underline{\varepsilon}$ are solutions of the cellular problem. The secant formulation of the homogenized thermo-hydro-mechanical behaviour law can be written as follows:

$\underline{\underline{\Sigma}}=C^{h o m}(\underline{\underline{E}}):\left(\underline{\underline{E}}-\underline{\underline{\alpha}}^{\text {hom }}(T) \Delta T\right)-p \underline{\underline{B}}$

where $C^{h o m}(\underline{E})$ is the homogenized stiffness tensor, $\underline{\alpha}^{\text {hom }}(T)$ the homogenized thermal expansion tensor, $p$ the average pressure and $\underline{B}$ the homogenized Biot's tensor. A direct estimation of this later tensor has been proposed by [45] and [46] from an estimation of the homogenized stiffness tensor $C^{\text {hom }}$ :

$\underline{\underline{B}}=\left(\underset{\sim}{I-C^{h o m}}:{\underset{\sim}{S}}^{S}\right): \underline{\underline{\delta}}$

where $S^{s}$ is the compliance tensor of the solid medium $\left(=1 / C^{s}\right), \underline{\delta}$ the second-order identity tensor and $I$ the fourth-order identity tensor. In the same way, the effective hydraulic properties can be calculated.

\subsection{The non-linear hydraulic behaviour}

Multiscale approaches can be also used to define the permeability of saturated or unsaturated porous media [47-51]. These works show that the fluid movement can be supposed Newtonian in small pores and satisfied Navier-Stokes equations. In neglecting friction in the fluid phase and inertial effects, the relation between the average of the local speed fields and pressures average leads to the Darcy's law [52]. That is why we have supposed in this study the hydraulic transport defined by the Darcy's law on the elementary volume scale. So, if a homogeneous macroscopic hydraulic load is applied on $\partial V$, a local speed field $\underline{v}(y)$ and a local hydraulic potential $h(y)$ are linked by the following behaviour law:

$\underline{v}(\underline{y})=-\underline{\underline{k}}(\underline{y}, \underline{\underline{\varepsilon}}(\underline{y}), T) \underline{\operatorname{grad}}(h(\underline{y})) \quad \underline{y} \in V$

where $\underline{k}(y, \underline{\varepsilon}(y), T)$ is the permeability tensor of phases (matrix or inclusion) depending on the local strains. Experimental tests on damaged cement-based materials have shown a modification of the microstructure and therefore a permeability variation. Under severe conditions (thermal and/or mechanical), the permeability of cement-based materials can greatly increase [53-56]. To take into account this phenomenon in the multiscale approach proposed in this work, the permeability of the concrete phases is supposed to be dependent on the local strain state. This model has been retained at the mesoscopic scale by supposing that all concrete phases are continuous at this scale. A permeability function, suggested by $\mathrm{J}$. Arsenault in [57], according to the volumetric damage has been retained for a continuous cement paste in concrete:

$\underline{\underline{k}}(\underline{y}, \underline{\underline{\varepsilon}}(\underline{y}))=k_{0}(\underline{y}) \cdot \exp \left(\kappa d^{s}\left(\tilde{\varepsilon}^{s}(\underline{y})\right)^{\beta}\right) \cdot \underline{\underline{\delta}}$

where $k_{0}(y)$ is the initial permeability coefficient of the local phase and $\kappa$ and $\beta$ are constant material parameters which have been calibrated to the material studied. This empirical relation has been obtained experimentally by considering concrete is homogenous. Picandet et al. [56] have shown the influence of $\kappa$ and $\beta$. In this work, these constant parameters have been calibrated for a highperformance concrete at high temperatures. In the temperature range studied here, the damage is preponderant in the cement paste. The evolution of the macroscopic permeability highly depends on the increase of the cement paste permeability. So, we consider the relation (9) is valid and active at mesoscale for the cement paste. The relation is not used for aggregate because we consider that aggregates are slightly porous and their damage is not sufficient to create a network flow until the temperature of $500{ }^{\circ} \mathrm{C}$.

It is supposed that the volumetric damage is responsible of the evolution of the capillary volume according to a hydrostatic strain, and that the deviatoric damage is only responsible of the orientation of the capillary network according to a shear strain.

A macroscopic speed $\underline{U}$ is applied through the following boundary condition:

$\underline{v}(\underline{y}) \cdot \underline{n}=\underline{U} \cdot \underline{n} \quad$ on $\quad \partial V$

where $\underline{n}$ is the outer normal vector of the area $V$.

The homogenized permeability tensor is then defined by the following relation between average fields:

$\langle\underline{v}(\underline{y})\rangle_{V}=-\underline{\underline{k}}^{h o m}(\underline{\underline{E}}, T)\langle\underline{\operatorname{grad}}(h(\underline{y}))\rangle_{V}$

\section{Application on the microscopic scale: study of cement pastes}

\subsection{The cement paste microstructure}

The Digital Concrete Model is used to generate the REV of the cement paste microstructure. The cement paste is supposed to be composed of two phases: the capillary porosity and a solid phase formed by the hydrated products and unhydrated cement particles. In the homogenization process, the solid inclusions are supposed to embed in the porosity. Here, the porosity plays the role usually taken by the matrix in the homogenization of composite materials, as a softer phase. Because it is difficult to simulate the smallest particles with diameter $<1 \mu \mathrm{m}$, we suppose these particles contained in the first phase. So, these particles give an elastic property of this phase, presented below. The generation of a REV of this material microstructure requires to firstly identify the volume fractions and the size distribution of each phase. This characterization is based on the cement paste formation during the hydration process which is determined by the Arrhenius law. The mechanical properties of the cement paste is then calculated by the self-consistent method according to the mechanical values of each constituents [58].

To simplify the calculation, in this paper, hydrated phases will be represented only by $\mathrm{C}-\mathrm{S}-\mathrm{H}$ and $\mathrm{CH}$ by considering negligible the other hydrated phases. They are given here at the end of the hydration process for various $w / c$ ratio in Table 1 . This calculation is based on cement pastes mixtures given in [59]. Then, the solid medium volume of cement pastes $V_{s}$ is given by the sum of the partial volume fraction $V_{r}$ of each cement particles and the porosity value $\phi$ with $\phi=1-V_{s} / V$. The cement particle size distribution is 
Table 1

Volume fraction of the cement pastes phases.

\begin{tabular}{lccc}
\hline$w / c$ & 0.3 & 0.4 & 0.5 \\
\hline$\alpha_{\text {hyd }}$ & 0.732 & 0.93 & 1 \\
$V_{\text {hyd }}(\%)$ & 80.3 & 87.8 & 82.9 \\
$V_{\mathrm{C}-\mathrm{S}-\mathrm{H}}(\%)$ & 58.2 & 63.7 & 60.1 \\
$V_{\mathrm{CH}}(\%)$ & 22.1 & 24.1 & 22.8 \\
$V_{\text {unhyd }}(\%)$ & 13.8 & 3.1 & 0 \\
$\phi(\%)$ & 0.059 & 0.091 & 0.171 \\
\hline
\end{tabular}

calculated by the Rosin-Rammler model according to experimental tests [60] (Table 2).

By considering this particle size distribution which not distinguish the inclusions type, each cement particle are now modeled by an assembly of an unhydrated particle surrounded by the hydrated products (Fig. 1 where $\mathrm{C}-\mathrm{S}-\mathrm{H}$ contains respectively the low and the high density $\mathrm{C}-\mathrm{S}-\mathrm{H}: \mathrm{C}-\mathrm{S}-\mathrm{H}_{a}$ and $\mathrm{C}-\mathrm{S}-\mathrm{H}_{b}$ with a ratio of $30-70 \%$ [61]).

\subsection{Mechanical behaviour of phases}

The solid inclusions formed by the main hydrated products ( $\mathrm{C}$ $\mathrm{S}-\mathrm{H}$ and $\mathrm{CH}$ ) and unhydrated cement particles are supposed to be elastic and isotropic. The local behaviour law is governed by the relation (1) where $p_{c}=0$ and $C(y)$ is constant with $d^{s}=0$ and $d^{d}=0$ (in relation (2)). The matrix formed by the capillary porosity containing the smallest cement particles (diameter $<1 \mu \mathrm{m}$ ) has a

Table 2

Volume fraction of solid inclusions for different cement pastes.

\begin{tabular}{rccc}
\hline$w / c$ Diameter $(\mu \mathrm{m})$ & $0.3 V_{r}(\%)$ & $0.4 V_{r}(\%)$ & $0.5 V_{r}(\%)$ \\
\hline 73 & 2.75 & 2.65 & 2.33 \\
61 & 5.70 & 5.51 & 4.84 \\
47 & 3.75 & 3.62 & 3.18 \\
41 & 4.88 & 4.71 & 4.14 \\
35 & 4.05 & 3.91 & 3.43 \\
31 & 2.31 & 2.23 & 1.96 \\
29 & 2.52 & 2.43 & 2.14 \\
27 & 2.75 & 2.66 & 2.33 \\
25 & 3.00 & 2.90 & 2.55 \\
23 & 3.28 & 3.17 & 2.78 \\
21 & 3.58 & 3.46 & 3.04 \\
19 & 3.91 & 3.78 & 3.32 \\
17 & 4.27 & 4.13 & 3.62 \\
15 & 4.66 & 4.51 & 3.96 \\
13 & 5.09 & 4.92 & 4.32 \\
11 & 5.56 & 5.37 & 4.72 \\
9 & 6.07 & 5.87 & 5.15 \\
7 & 6.63 & 6.41 & 5.62 \\
5 & 7.24 & 7.00 & 6.14 \\
3 & 12.13 & 11.72 & 10.29
\end{tabular}

small stiffness with a shear modulus $\mu$ taken arbitrarily equal to $1 \times 10^{-5} \mathrm{MPa}$. So, its behaviour is essentially governed by the capillary pressure $p_{c}$. In addition, we suppose that the fluid sweeps the pore surface and the interface pressure in pores between the solid medium and the fluid phase is taken equal to zero [62]. The behaviour law of the capillary network is taken as follow:

$\underline{\underline{\sigma}}(\underline{y})=-p_{c} \underline{\underline{\delta}}+2 \mu \underline{\underline{\varepsilon}}(\underline{y})$

where $p_{c}$ is calculated by the Laplace's law:

$p_{c}-p_{0}=\frac{R \cdot T}{v_{w}} \cdot \ln \left(R_{h}\right)$

where $R\left(=8.314 \mathrm{~J} \mathrm{~K}^{-1} \mathrm{~mol}^{-1}\right)$ is the perfect gas constant, $T$ $\left(=293.15^{\circ} \mathrm{K}\right)$ the absolute temperature, $v_{w}\left(=1.8 \times 10^{-5} \mathrm{~m}^{3} \mathrm{~mol}^{-1}\right)$ the specific molar volume of water, $p_{0}(=0.1 \mathrm{MPa})$ the reference pressure and $R_{h}$ the internal relative humidity. In this study, $R_{h}$ is supposed to be constant and arbitrarily equal to $95 \%$.

The mechanical properties of the solid inclusions are obtained by two successive homogenization calculations by applying the Mori-Tanaka method (Fig. 1). The homogenized Young's moduli and Poisson's ratios of the solid medium have been obtained for different $w / c$ ratios and are presented in Table 3 .

\subsection{Hydraulic properties of cement paste phases}

In the same way, the local hydraulic properties are calculated. The hydraulic behaviour is taken linear for each phases. The local permeability tensor $k(y, \underline{\varepsilon}(y))$, defined in the relation (8), is taken constant and equal to $k_{h}$ in the capillary network and $k_{l}$ in the solid phase. These permeability coefficients are deduced from the works of Bentz and Garboczi $[35,63]$ based on the Katz-Thompson model [64]. The solid part of the cement paste is a low permeable phase and by exploiting the Katz-Thompson relation, its permeability can be taken equal to [59]:

$k_{l}=k_{\mathrm{CSH}}\left[1-\frac{1-f_{\mathrm{CSH}}(w / c)}{1-f_{c}^{\prime}}\right]^{2}$

where $f_{c}^{\prime}=0.17$ [35] is the critical volume fraction of C-S-H obtained by percolation, $f_{\mathrm{C}-\mathrm{S}-\mathrm{H}}$ the volume fraction of $\mathrm{C}-\mathrm{S}-\mathrm{H}$ in the solid phase and $k_{\mathrm{C}-\mathrm{S}-\mathrm{H}}=7 \times 10^{-16} \mathrm{~m} \mathrm{~s}^{-1}$ [65] the water permeability of $\mathrm{C}-\mathrm{S}-\mathrm{H}$ pore gels.

Table 3

Calculated elastic properties of the solid particles.

\begin{tabular}{lll}
\hline$w / c$ & $E(\mathrm{GPa})$ & $v(-)$ \\
\hline 0.3 & 32 & 0.25 \\
0.4 & 27.6 & 0.255 \\
0.5 & 26.5 & 0.257 \\
\hline
\end{tabular}

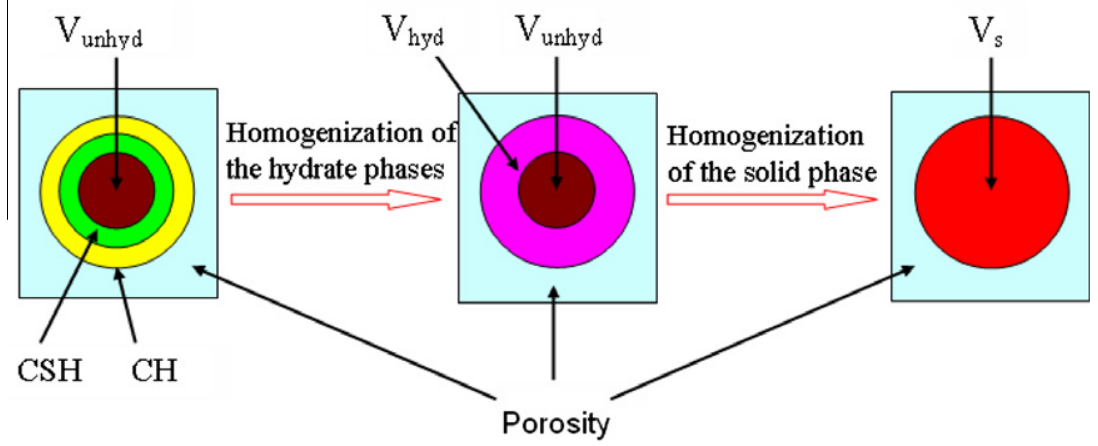

Fig. 1. Homogenization of the solid medium. 
The capillary network is a high-permeable phase and, by identifying to the Katz-Thompson relation, its permeability is written as follow:

$k_{h}=\frac{1.8}{226} l_{c}^{2}\left(1-f_{c}\right)^{2}$

where $f_{c}=0.18$ [65] is the volume fraction of the porosity if a connectivity exists and $l_{c}$ the critical pore radius which is the pore radius assuming a connectivity of the capillary network. $l_{c}$ is obtained by measuring the pore size distribution and it corresponds to the first peak in the logarithm curve for pore radius greater than $1 \mathrm{~nm}$. In this study, the permeability has been calculated at the end of the hydration process, because the Katz-Thompson model has not been performed for cement-based materials at early age. According to the relations (14) and (15), the permeability of the capillary network $k_{h}$ and the permeability of the solid medium of the cement paste $k_{l}$ are given for different cement pastes in Table 4 .

\subsection{Equivalent mechanical and hydraulic behaviour of cement pastes}

The effective Young's modulus and the Poisson's ratio of different cement pastes have been calculated by solving cellular problems (1) with the Digital Concrete model and the F.E. code Symphonie. Calculated Young's moduli are compared in a good agreement to experimental measurements $[66,67]$ for various $w / c$ ratios (Fig. 2). The calculation of the homogenized Poisson's ratios in two main directions shows the possibility of the model to reproduce the isotropic behaviour of a cement paste (Table 5).

Table 4

Calculated permeability of the cement pastes phases at 210 days.

\begin{tabular}{lll}
\hline$w / c$ & $k_{l}\left(\mathrm{~m}^{2}\right)$ & $k_{h}\left(\mathrm{~m}^{2}\right)$ \\
\hline 0.3 & $2.04 \times 10^{-23}$ & $5.46 \times 10^{-19}$ \\
0.4 & $2.85 \times 10^{-23}$ & $5.46 \times 10^{-19}$ \\
0.5 & $2.93 \times 10^{-23}$ & $5.46 \times 10^{-19}$ \\
\hline
\end{tabular}

Table 5

Poisson's coefficients of different cement pastes.

\begin{tabular}{lll}
\hline$w / c$ & $v_{x x}$ & $v_{y y}$ \\
\hline 0.3 & 0.247 & 0.248 \\
0.4 & 0.252 & 0.25 \\
0.5 & 0.254 & 0.256 \\
\hline
\end{tabular}

The equivalent permeability is also compared in a good agreement to experimental tests [59] for two $w / c$ ratios, 0.3 and 0.4 (Table 6). The permeability depends on the cement composition and the maturity of the cement paste. In Fig. 3 the ratio $K / K_{0.3}$ between the calculated permeability of different $w / c$ ratios on that of a cement paste with $w / c=0.3$ is presented. For a high $w / c$ ratio, the water permeability takes a very high value. The evolution of the water permeability with $w / c$ can be represented following an exponential curve. Similar results are observed in experimental tests [68]. In experimental procedures, before measuring the permeability, the cement paste is dried (until to $105^{\circ} \mathrm{C}$ ) in order to unsaturate the capillary pores. But there is always a small volume of residual water after drying which influences the hydraulic flow during the permeability test. In the calculation, we have supposed that pores do not contain water. Results could be improved by taking into account the hydration process of each cement particles and to take into account the residual water in the capillary porosity. Such developments are in progress [58].

Table 6

Comparisons between calculated results and experimental tests for the equivalent intrinsic permeability of cement pastes.

\begin{tabular}{lll}
\hline$w / c$ & Simulation $\left(\mathrm{m}^{2}\right)$ & Tests [59] $\left(\mathrm{m}^{2}\right)$ \\
\hline 0.3 & $2.46 \times 10^{-23}$ & $<2.69 \times 10^{-23}$ \\
0.4 & $4.07 \times 10^{-23}$ & $12.2 \times 10^{-23}$ \\
\hline
\end{tabular}

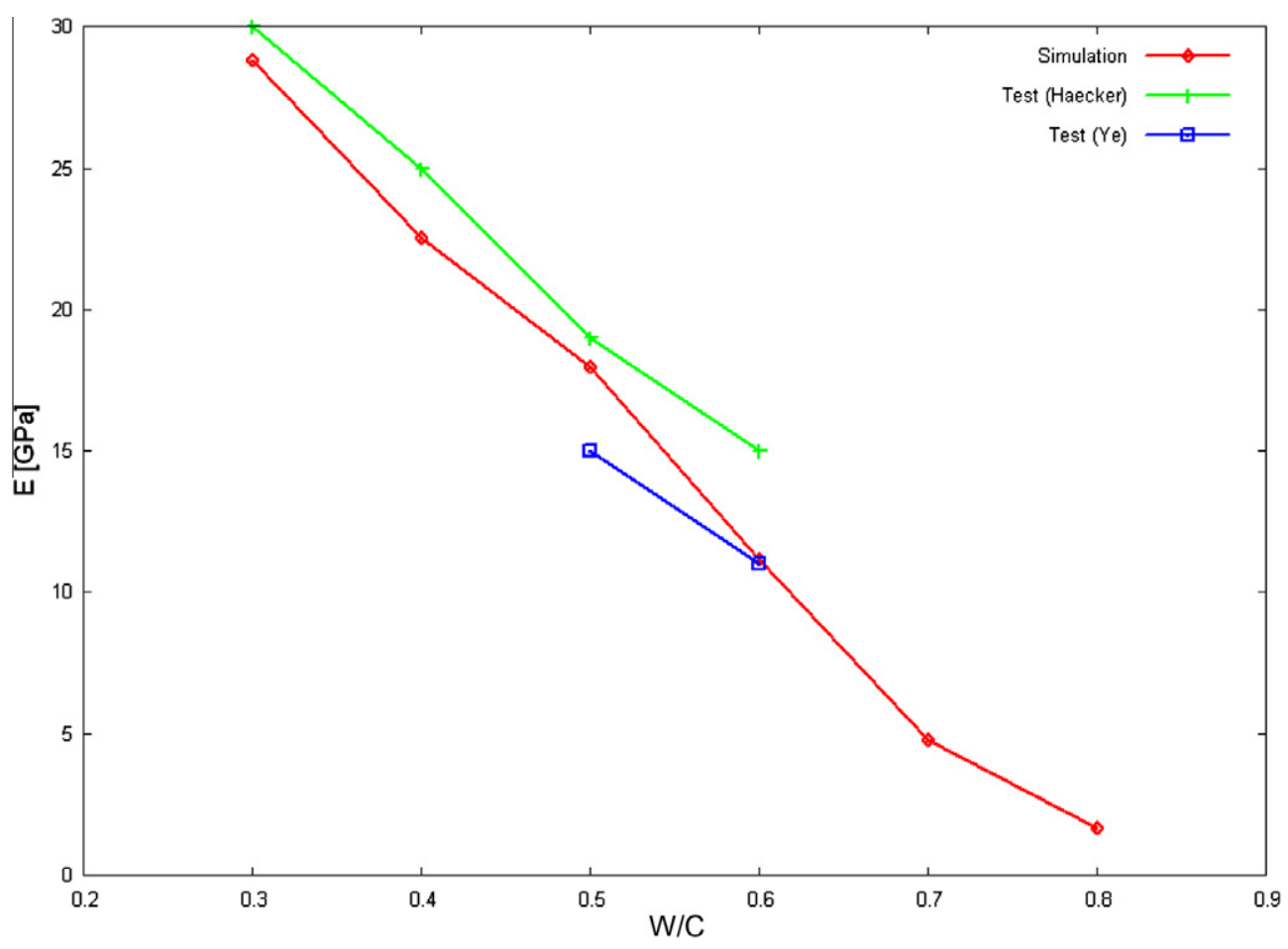

Fig. 2. Comparison of effective calculated Young moduli and experimental measurements for different $w / c$ at 210 days. 




Fig. 3. Relationships between calculated intrinsic permeability and $w / c$ for mature cement pastes.

\subsection{Equivalent hydro-mechanical behaviour of cement pastes}

The behaviour of porous media are governed by a lot of physical and mechanical parameters. Their experimental characterization is sometimes difficult according to their coupled evolution [69]. Among these macroscopic parameters, the hydraulic expansion coefficient, also called Biot's modulus $M$ [70], is an example of these multi-physics parameters difficult to identify experimentally. See for instance the work of Obeid and Mounajed [41] for cement mortars. Therefore, the determination of the hydro- mechanical behaviour of porous media requires the knowledge of $\underline{B}$. The Biot's modulus $M$ is then defined by the following relation:

$\frac{1}{M}=\frac{b-\phi}{K_{s}}+\frac{\phi}{K_{f l}}$

where $K_{s}(\mathrm{MPa})$ is the compressive modulus of the solid medium, $K_{f l}$ (MPa) the compressive modulus of the fluid, $\phi$ the porosity and $b$ the Biot's coefficient used for an isotropic material as $\underline{\underline{B}}=b \underline{\underline{\delta}}$. The Biot's coefficient represents the fraction of the volume variation of the material corresponding to the volume variation of the pore

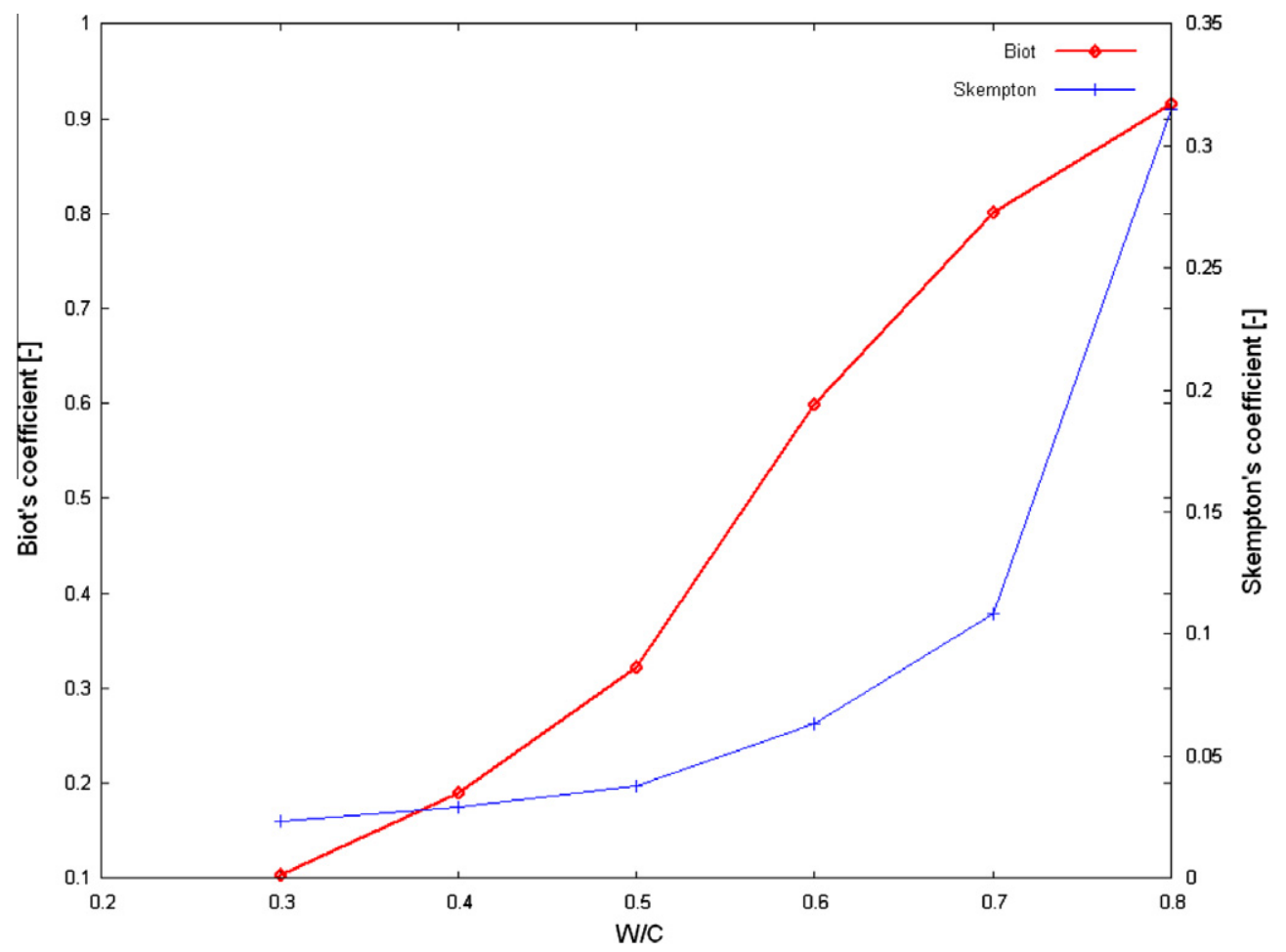

Fig. 4. The Biot's coefficient and the Skempton coefficient versus $w / c$. 
space. Its expression can be generalized to several fluids in the pore space [41]. Under the same conditions, the Skempton's coefficient $B_{s}$, which represents the ratio of the pore pressure increment to the mean stress increment under undrained conditions, is written as follow:

$\frac{1}{B_{s}}=\frac{K_{0}}{M b}+b$

where $K_{0}$ is the compressive modulus of the drained material.

By using the relation (7), the Biot's tensor is calculated according to the homogenized stiffness tensor calculated below and the compliance tensor of the solid cement particles. The calculated Biot's tensor is isotropic and its value increases with the $w / c$ ratio according to a higher capillary porosity. Calculated results of the

Table 7

The aggregate size distribution of concrete.

\begin{tabular}{lclc}
\hline$D(\mathrm{~mm})$ & $V_{f}(\%)$ & $D(\mathrm{~mm})$ & $V_{f}(\%)$ \\
\hline 25 & 2 & 4 & 3 \\
20 & 8 & 3.15 & 1 \\
16 & 10 & 2.5 & 3 \\
12.5 & 8 & 2 & 1 \\
10 & 6 & 1.6 & 1 \\
8 & 4 & 1.25 & 4 \\
6.3 & 2 & 1 & 18 \\
5 & 2 & & \\
\hline
\end{tabular}

Table 8

Initial mechanical parameters of the concrete phases.

\begin{tabular}{lll}
\hline Exp. values & Cement paste & Aggregate \\
\hline$E(\mathrm{GPa})$ & 15 & 75 \\
$v$ & 0.2 & 0.28 \\
$f_{c}(\mathrm{MPa})$ & 80 & 180 \\
$G(\mathrm{~N} / \mathrm{mm})$ & 0.1 & 0.15 \\
Calibrated values & & \\
$f_{t}(\mathrm{MPa})$ & 3 & 10 \\
$f_{s}(\mathrm{MPa})$ & 6 & 20 \\
\hline
\end{tabular}

Biot's coefficient and the Skempton's coefficient for different cement pastes are presented in Fig. 4. Calculated values for $w / c$ ratios equal to $0.3,0.4$ and 0.5 are around 0.2 . It is a good result in comparison to experimental values measured for ordinary cementbased materials (with $w / c<0.5$ ) which are unsaturated with a relative humidity greater than $80 \%$ [41].

\section{Application on the mesoscopic scale: a high-performance concrete in tension at high temperatures}

\subsection{The concrete components properties and the loading}

Applications concern the behaviour of a High Strength Concrete studied in experimental fire tests [19,71]. Tested concrete have been mixed with a Portland cement (type CPA CEM I 52.5 PM ES CP2 of Le Havre) with a $w / c$ ratio equal to 0.3 , crashed limestone aggregate $0 / 20$ (Boulonnais) and sand-lime 0/4 (La Seine). The aggregate size distribution is presented in Table 7 where $V_{f}$ is the volume fraction of each aggregate in \% and $D$ their diameter in $\mathrm{mm}$. In the experimental tests, cylindrical concrete specimens of size $\phi 16 \times 32 \mathrm{~cm}^{2}$ have been tested.

The mechanical properties of the concrete phases at 28 days are given by experimental measurements $[72,19,8,73]$ and are summarized in Table 8 where $f_{c}$ is the compressive strength. $f_{t}$ and $f_{s}$ have been identified by an inverse method on a representative elementary volume under a constant unit mechanical load. The thermal expansion coefficient of the cement paste and of aggregate are given by experimental measurements [2,19] and are presented in Fig. 5.

A uniform tensile load combined with a uniform temperature $\Delta T$ are applied on the specimen in the same conditions as the experiments. The initial tensile strength of the material is given equal to $5 \mathrm{MPa}$ and the tensile load (TL) applied on the concrete specimen is taken equal to $0 \mathrm{MPa}, 1 \mathrm{MPa}$ and $2 \mathrm{MPa}$ which correspond respectively to a load-to-strength $(L / S)$ ratio equal to $0 \%$, $20 \%$ or $40 \%$. The rate of temperature is equal to $1{ }^{\circ} \mathrm{C} / \mathrm{min}$ and the test starts at $20^{\circ} \mathrm{C}$ up to $600{ }^{\circ} \mathrm{C}$. To make strain measurements at high temperatures, Hager and Pimienta [19] have retained an ori-

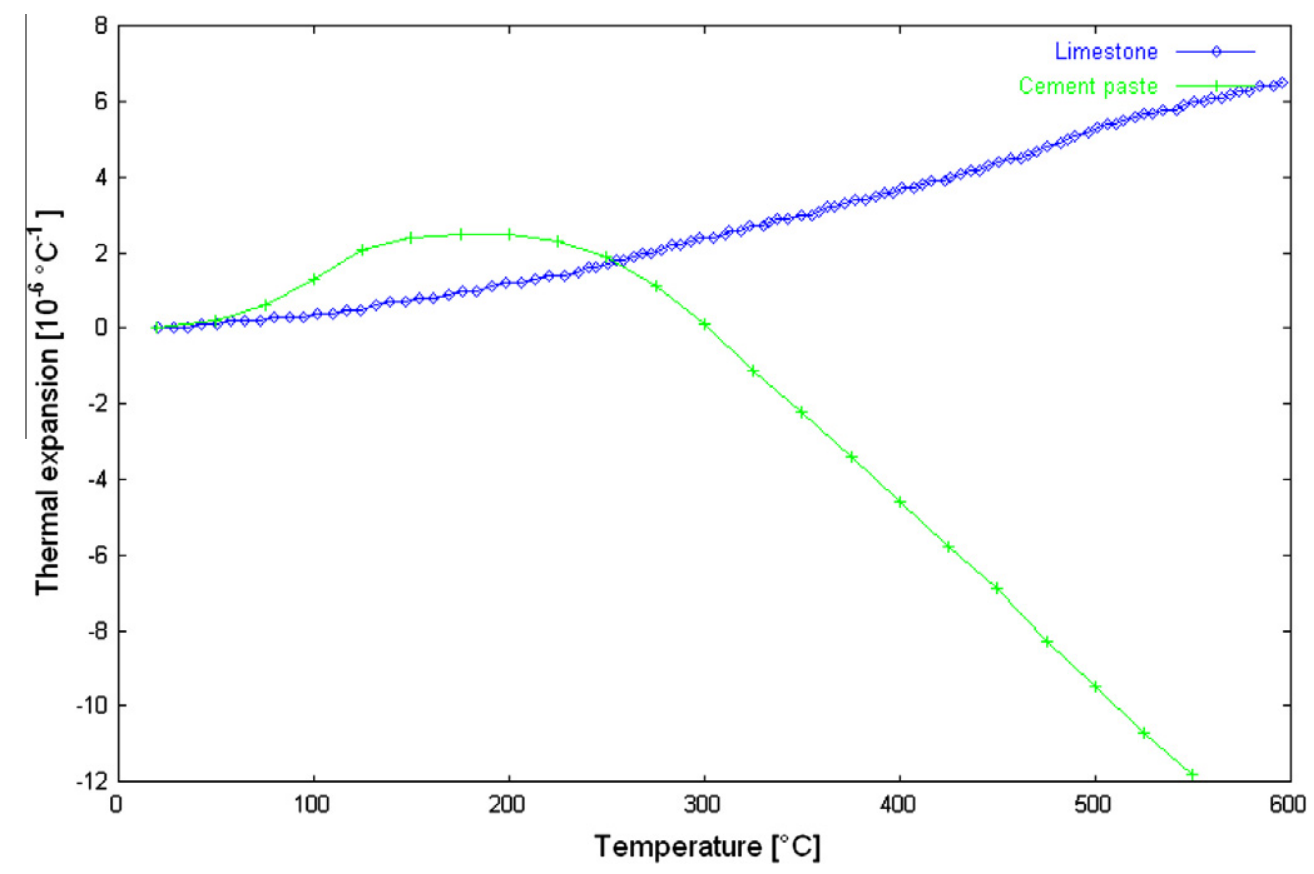

Fig. 5. Evolution of the thermal expansion of the cement paste and limestone aggregate versus temperature. 
ginal method, called 'lateral grip': metal jaws are fixed at the concrete cylinder tips. But the authors have been observed that the clamping develops a biaxial stress state which can lead to damage of the concrete cylinder tips. They have neglected this damage in measurements and have supposed that damage occurs only in the centre of the specimen.

In experimental tests, why the speed of temperature increasing was $1^{\circ} \mathrm{C} / \mathrm{min}$, no pressure gradient occured [8]. That is why in simulations, the speed of temperature increasing is not taken into account. So, the temperature is not transient. Also, in simulation we have considered material parameters for drying concrete as suggested in the experiments to prevent the influence of the gas pressure during the temperature increasing [19]. Hager and Pimienta have shown that drying at $105^{\circ} \mathrm{C}$ to have a total moisture-loss leads to a decrease of the gas pressure from $4 \mathrm{MPa}$ to $0.28 \mathrm{MPa}$. So, the capillary pressure in relation ( 1 ) is taken equal to 0 , by supposing that the pressure is not influent on damage.

\subsection{Strain and damage of concrete}

The REV is computed in two dimensions and a plane strain condition is assumed. It is computed by taking into account the real aggregate size distribution of concrete (Table 7). The volume dimensions are chosen according to the ratio with the maximum aggregate diameter. The validity of this two dimensional simulation has been discussed in previous work [15]. Effective mechanical characteristics obtained by this approximation are in good agreement with experimental data or classical 3D models. Elsewhere, the interfacial transition zone (ITZ) is not modelled because it has been observed that the chemical bonds between crushed limestone aggregates and the cement paste are strong [74]. So the interface between the cement paste and the aggregates is supposed to be perfect in the model and concrete can be modelled by only two phases.

The macroscopic loads are applied in the REV boundary in the same conditions as the experimental tests where $U_{x}$, respectively $U_{y}$, is the horizontal displacement, respectively the vertical displacement, $T$ the temperature imposed in the boundaries and $F$ the tensile load (Fig. 6). In this work, three configurations have

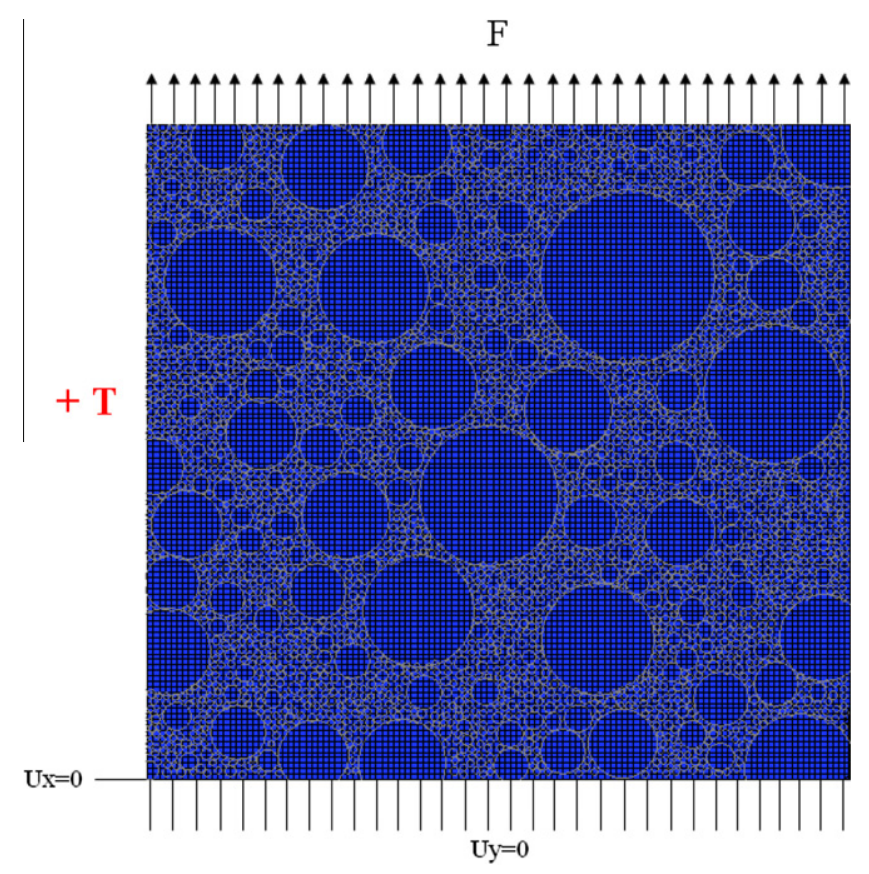

Fig. 6. The Digital Concrete REV and the boundary conditions. been used and, as explained in [15], homogenized elastic properties do not vary with the configuration. In the previous paper [15], we have shown that the REV configuration does not influence the macroscopic strain values until the temperature of $500^{\circ} \mathrm{C}$, however damage has nucleated just before $100^{\circ} \mathrm{C}$. Up to $500^{\circ} \mathrm{C}$, low damage occur in the aggregate surfaces and beyond this temperature they begin to fail, but the model does not take into account this failure mechanism. In the case of tensile load with temperature, damage begins before $100{ }^{\circ} \mathrm{C}$ whatever the tested tensile load. The difference of results for different geometries is not significant in comparison with the computer relative error and with the experimental tests variability. The local strain fields are induced by the macroscopic loading. Calculated results for the strain evolution are compared to experimental measurements in Fig. 7. The strain evolutions with the temperature for the ratio $L /$ $S=20 \%$ and $L / S=40 \%$ are very close to each other. It can be explained by the fact that in tension (with or without temperature) deformation evolves similarly in concrete. Therefore, differences are observed for the time of failure. The load force has no influence on the shape of the strain curve but only on the total failure which appears rapidly for $L / S=40 \%$.

We have shown that the random distribution of inclusions has an influence on the time of failure of concrete in compression at high temperatures in a recent work [15]. So it would be interesting to model different geometries to analyze the influence of the random microstructure on the degradation due to a tensile load. In this study, we focus on the stiffness degradation and on the increase of the permeability of the damaged material.

The strength of concrete at high temperatures decreases due to local damage in the microstructure [15]. Concrete has a low tensile strength. Therefore, in experimental tests it is very difficult to measure the Young's modulus of concrete which have been submitted to high temperatures and to a tensile load. There are very few results about this subject in the literature review. The concrete specimens used by Felicetti and Gambarova [18] have respectively tensile and compressive strengths close to that presented in this paper, respectively $4.5 \mathrm{MPa}$ and $5.5 \mathrm{MPa}$ for $f_{t}$ and $72 \mathrm{MPa}$ and $95 \mathrm{MPa}$ for $f_{c}$. Calculated results are presented in Fig. 8. For $L /$ $S=20 \%$ Felicetti and Gambarova have obtained a residual elastic modulus equal to $70 \%$ of its initial value at $250{ }^{\circ} \mathrm{C}$. Which is similar to calculated results obtained in this work.

\subsection{Relations between damage and permeability}

The macroscopic permeability is explicitly dependent on the damage value. Because the brittle zone of the material (the cement paste) has a low surface, the influence of the REV configurations is not significant on the macroscopic permeability as for the macroscopic strain. As for the Young modulus, experimental measurements are limited by the fragile behaviour of concrete at high temperatures under a tensile load. In fact, no results have been found in the literature review. No devices to measure the permeability under these conditions exist. That is the interest of the development of a modelling approach.

Calculated results are presented in Fig. 9. Results obtained for $L /$ $S=0 \%$ are in a good agreement to experimental measurements $[75,76]$. The evolution of the effective permeability (which corresponds to the intrinsic permeability) seems to follow the evolution of the macroscopic total strain observed on Fig. 7. So, the evolution of the permeability is similar in the two tests $(L / S=20 \%$ and $L /$ $S=40 \%$ ). In comparison to calculations performed on the same material at high temperatures under a compressive load [15], the permeability increases more rapidly in the tensile case. Physically, it can be explained by the separation of the CSH layers (formed during the cement hydration) imposed by the thermal expansion of the cement paste and by the tensile load. 




Fig. 7. Comparison of calculated and measured total strains of concrete at high temperatures for $L / S=20 \%$ and $L / S=40 \%$.

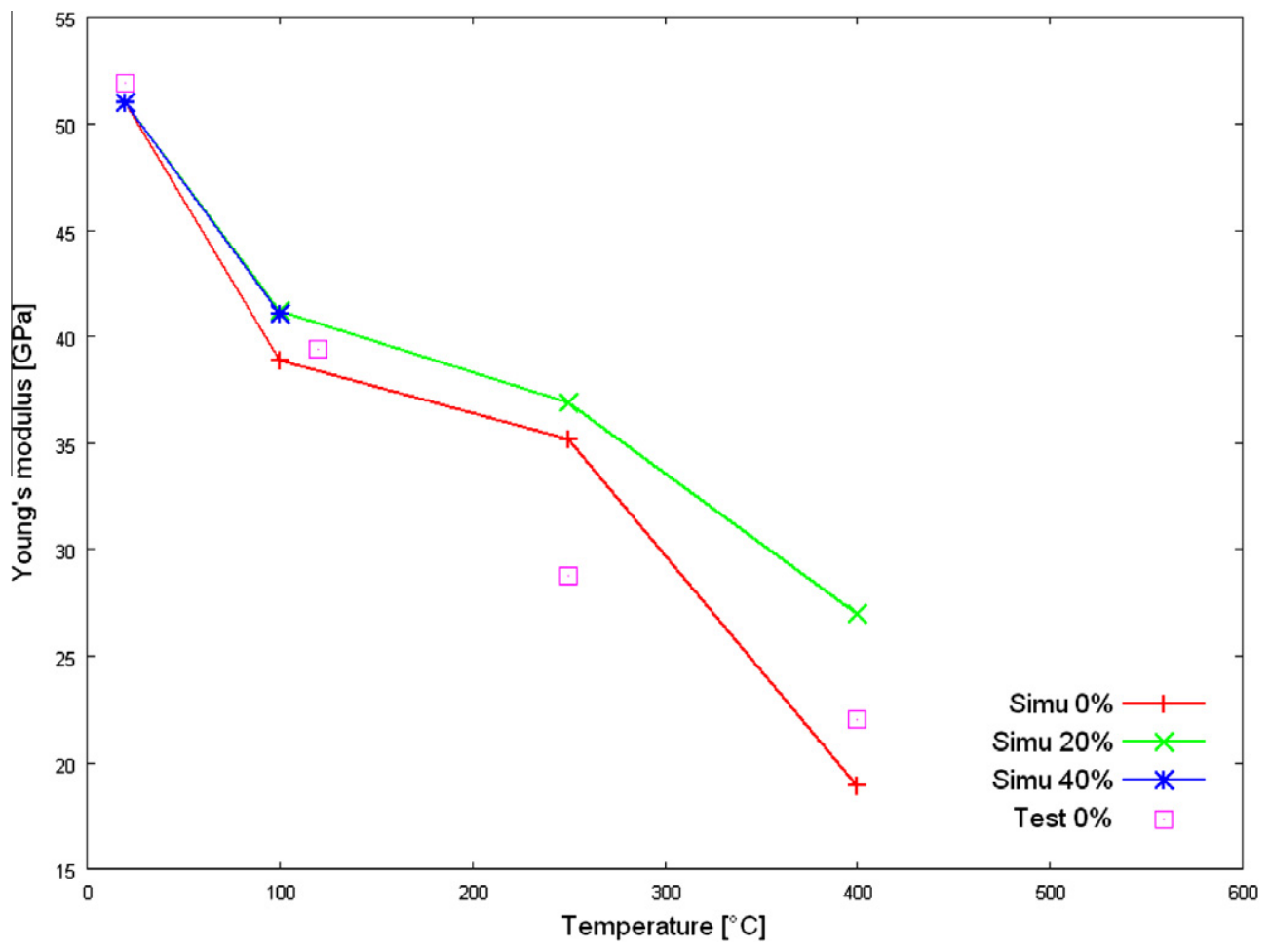

Fig. 8. Calculated results for the Young's modulus of damaged concrete.

After these two calculations, mechanical and hydraulic results obtained can be interpreted as follow. The total strain seems to be mainly due to the thermal expansion which contributes to the separation of the $\mathrm{C}-\mathrm{S}-\mathrm{H}$ layers. And, the tensile load leads to the total failure of the material, but not contributes, or very few, to the strain evolution.

\subsection{The micromechanics analysis}

In recent works, we have showed the influence of the microstructure geometry on the deterioration of concrete at high temperatures under a mechanical load [15]. In this study the loss of rigidity of concrete at high temperatures in tension is attributed 


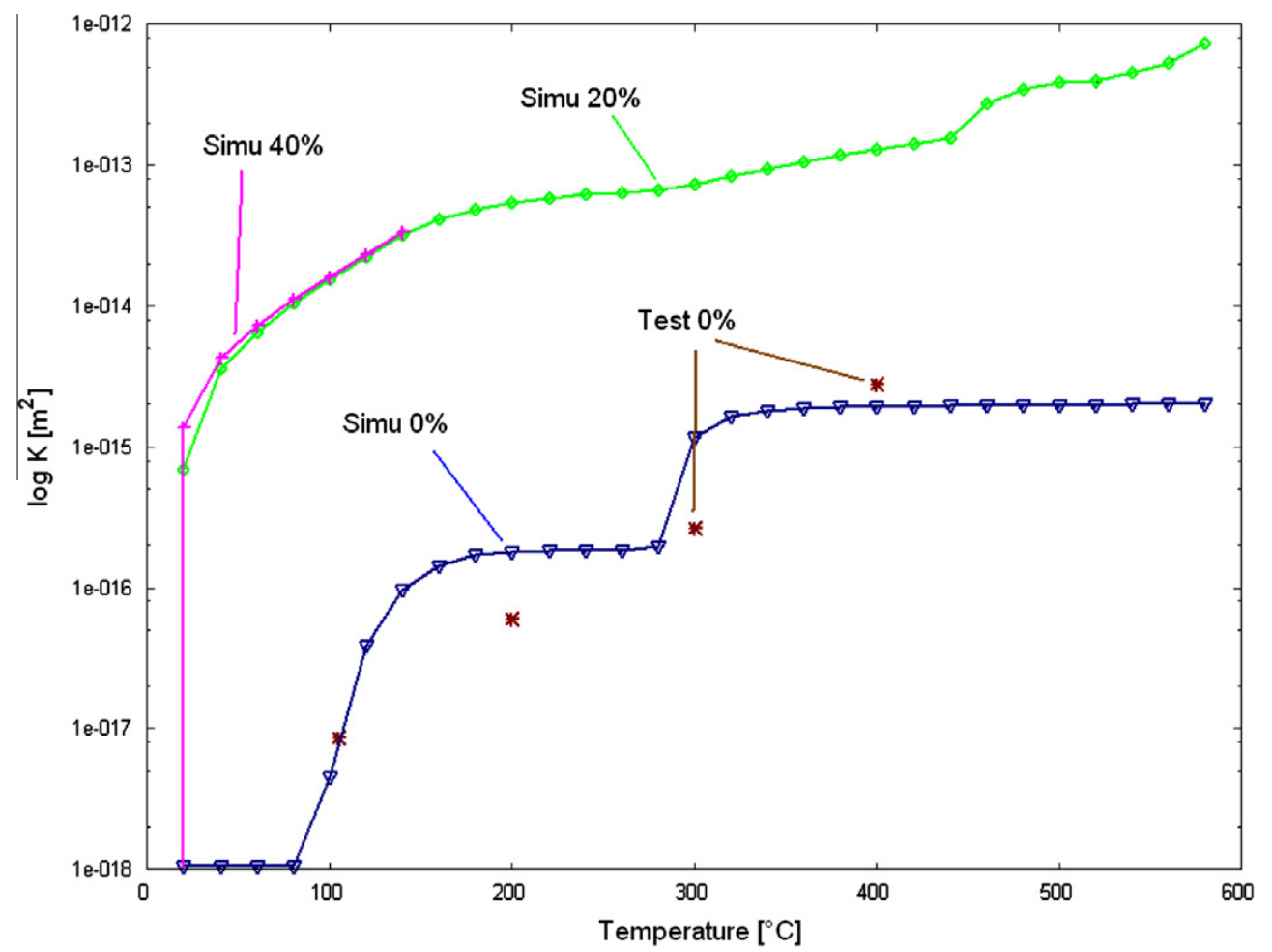

Fig. 9. The permeability evolution of concrete at high temperatures under a tensile load.


Fig. 10. Localization of damage in concrete at $100^{\circ} \mathrm{C}$ and $140{ }^{\circ} \mathrm{C}$ for $L / S=40 \%$.
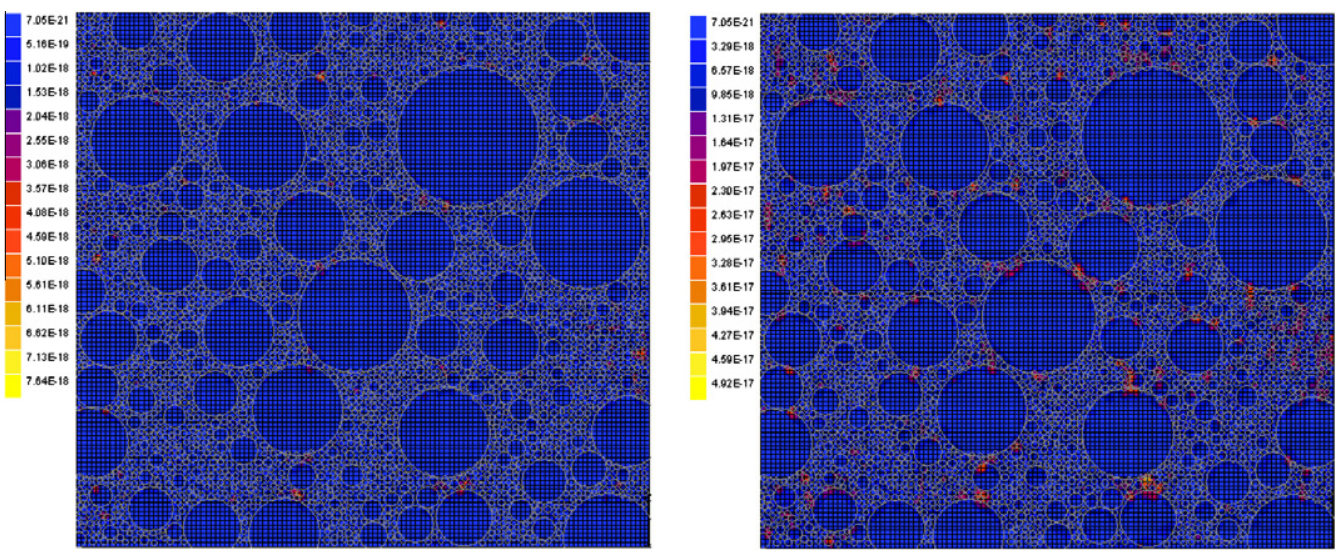

Fig. 11. Localization of flux in concrete at $100{ }^{\circ} \mathrm{C}$ and $140{ }^{\circ} \mathrm{C}$ for $L / S=40 \%$. 
to the degradation of the microstructure too. Fig. 10 shows the damage localization in the microstructure of concrete at $100{ }^{\circ} \mathrm{C}$ and $140{ }^{\circ} \mathrm{C}$. The damage level is defined by colors which change between blue (no damage) to yellow (cracked). Fig. 10 clearly shows higher damage in the cement paste at $140{ }^{\circ} \mathrm{C}$. The aggregates are less affected. And the damage average value is around 0.5 which is sufficient to increase the local porosity. At the same time, we observe the local hydraulic flux in Fig. 11. The effective permeability is the average of the local permeability. And it confirms results presented below on the increase of the effective permeability (Fig. 9).

\section{Conclusions}

In this paper, we have presented a numerical multiscale approach to model the thermo-hydro-mechanical behaviour of cement -based materials. A coupling of a homogenization method with the Digital Concrete Model of the finite element code Symphonie has been performed. The Digital Concrete Model allows to generate, following a random process, all heterogeneities in a Representative Elementary Volume. The volume geometry approaches the reality of the material microstructure. The homogenized behaviour laws of the equivalent material are obtained as the relation of the local fields averages calculated by the finite element method.

First of all, applications have been made on the determination of the mechanical, poro-mechanical and hydraulic properties of cement pastes with different water-to-cement ratios. Calculated results have been compared and are in good agreement with experimental measurements.

Secondly, analyses have been made to study the damage behaviour of concrete at high temperatures under a tensile load. Regarding the compressive behaviour at high temperatures, damage nucleates in the concrete microstructure due to differential dilations of the cement paste and aggregate [15]. Multiscale approaches seem to be well suited to model the behaviour of concrete under these conditions. Then, the calculation of the permeability of the damaged material has been computed. Calculations of the equivalent thermo-mechanical properties and the equivalent permeability are in a good agreement with experimental measurements. The specificity of this numerical approach is to follow the evolutions of local fields. The localization of the strains, respectively the hydraulic flows, has been allowed to follow the influence of the evolution of the porosity on the macroscopic mechanical behaviour, respectively on the effective permeability. We have observed an influence of the microstructure of concrete on the deterioration. The validity of this two dimensional simulation has been discussed in previous work [15]. Effective mechanical characteristics obtained by this approximation are in good agreement with experimental data.

In future works, we will implement diverse physico-mechanical responses of cement-based materials under different conditions, such as the self-dessication of concrete at early age [58], the chemical deterioration (external sulfate attacks, lixiviation, chloride ingress, etc.) [77] and the creep behaviour. To complete the model, we expect to implement the interfacial transition zone between aggregate and cement paste in concrete. To take into account the influence of crack shape on the permeability, we expect to implement a mathematical formulation of this relation [78]. Furthermore, calculations similar to those presented in this study are in course in three dimensions to show the influence of geometry on the computed results.

\section{References}

[1] Barragan B, Di Maio A, Giaccio G, Traversa L, Zerbino R, et al. Effects of high temperature on residual, mechanical, and transport properties of concrete. Am Concr Inst 2000;192:983-1000.
[2] Bazant Z, Kaplan M. Concrete at high temperatures: material properties and mathematical models. In: Concrete design and construction series. Longman Group Limited; 1996.

[3] Khoury G, Grainger B, Sulivan G. Transient thermal strain of concrete: literature review, conditions with specimen and behaviour of individual constituents. Mag Concr Res 1985;132:131-44

[4] Noumowe AN, Clastres P, Debicki G, Bolvin M. High temperature effect on high performance concrete $\left(70-600^{\circ} \mathrm{C}\right)$ strength and porosity. Am Concr Inst $1994 ; 145: 157-72$

[5] Committee RT. Recommendation of rilem tc 200-htc: mechanical concrete properties at high temperatures-modelling and applications. Part 1: introduction-general presentation. Mater Struct 2007;40(9):841-53.

[6] Committee RT. Recommendation of rilem tc 200-htc: mechanical concrete properties at high temperatures-modelling and applications. Part 2: stressstrain relation. Mater Struct 2007;40(9):855-64.

[7] Alonso C, Andrade E, Menendez C, Gayo E. Microstructural changes in high and ultra high strength concrete exposed to high temperature environments. Am Concr Inst 2005;229:289-302.

[8] Menou A, Mounajed G, Boussa H, Pineaud A, Carre H. Residual fracture energy of cement paste, mortar and concrete subject to high temperature. Theor Appl Fract Mech 2006;45:6471.

[9] Parrot L. Study of transitional thermal creep in hardened cement paste. Mag Concr Res 1979;31(107):99-103.

[10] Anderberg Y, Thelandersson S. Stress and deformation characteristics of concrete at elevated temperatures. Part II: experimental investigation and material behaviour model, Bulletin 54, Lund Institute of Technology; 1976.

[11] Sabeur H, Meftah F. A thermo-hydro-damage model for the dehydration creep of concrete subjected to high temperature. In: 3rd European conference on computational mechanics; 2006.

[12] Bazant Z, Cusatis G, Cedolin L. Temperature effect on concrete creep modeled by microprestress-solidification theory. J Eng Mech 2004;130(6):691-9.

[13] Gawin D, Pesavento F, Schrefler B. Modelling of deformations of high strength concrete at elevated temperatures. Mater Struct 2004;37(268):218-36.

[14] Mounajed G, Boussa H, Grondin F, Menou A. Transient thermal creep of concrete: intrinsic behaviour or structural effect? In: 7th Concreep, Nantes, France; 2005.

[15] Grondin F, Dumontet H, Ben Hamida A, Mounajed G, Boussa H. Multi-scales modelling for the behaviour of damaged concrete. Cem Concr Res 2007;37:1453-62.

[16] Menou A, Mounajed G, Boussa H, La Borderie C, Lafdi K. Thermal damage approach of concrete: application to specimens subjected to combined compressive and high temperature loads. J High Temp Mater Process 2008;27(1):23-40.

[17] La Borderie C, Lawrence C, Menou A. Approche mésoscopique du comportement du béton. Eur J Environ Civil Eng 2007;11(4):407-21.

[18] Felicetti R, Gambarova P. On the residual tensile properties of high performance siliceous concrete exposed to high temperature. In: Mechanics of quasi-brittle materials and structures. Hermes Sciences Publications; 1999.

[19] Hager I, Pimienta P. Déformation thermique transitoire des bétons à haute performance: Etude expérimentale. Rev Eur Génie Civil 2005;9(3): 373-383.

[20] Thelandersson S. Effect of high temperature on tensile strength of concrete. Tech rep 27, division of structural mechanics and concrete construction, Institute of Technology, Neostyled; 1971.

[21] Coussy O. Mechanics of porous continua. Wiley and Sons; 1995.

[22] Schrefler B. Computer modelling in environmental geomechanics. Comput Struct 2001;79:2209-23.

[23] Heinfling G, Reynouard J, Merabet O, Duval C. Thermo-elastic-plastic model for concrete at elevated temperatures including cracking and thermomechanical interaction strains. In: Owen DR, Onte E, Hinton E, editors. Computational plasticity: fundamentals and applications, Barcelona, Spain; 1997. p. $1493-8$.

[24] Luccioni B, Figueroa M, Danesi R. Thermo-mechanic model for concrete exposed to elevated temperatures. Eng Struct 2003;25:729-42.

[25] Mounajed G, Obeid W. A new coupling fe model for the simulation of thermalhydro-mechanical behavior of concretes at high temperatures. Mater Struct 2004;37(270):422-32.

[26] Nechnech W, Meftah F, Reynouard J. An elasto-plastic damage model for plain concrete subjected to high temperatures. Eng Struct 2002;24: 597-611.

[27] Chatzigeorgiou G, Picandet V, Khelidj A, Pijaudier-Cabot G. Coupling between progressive damage and permeability of concrete: analysis with a discrete model. Int J Numer Anal Methods Geomech 2005;29:10051018.

[28] Gawin D, Pesavento F, Schrefler B. Modelling of thermo-mechanical and mechanical damage of concrete at high temperature. Comput Methods Appl Mech Eng 2003;192:1731-71.

[29] Nechnech W, Reynouard J, Meftah F. On modelling of thermo-mechanical concrete for the finite element analysis of structures submitted to elevated temperatures. In: de Borst R, Mazars J, Pijaudier-Cabot G, van Mier J, editors. Proceedings of fracture mechanics of concrete structures, Swets and Zeitlinger, Lisse; 2001. p. 271-8.

[30] Ulm F-J, Acker P, Levy M. The chunnel fire. II. Analysis of concrete damage. ASCE J Eng Mech 1999;3:283-9.

[31] Zouari R, Dumontet H, Ben Hamida A. A micromechanical iterative approach for the behavior of poly-dispersed materials. Int J Solids Struct 2008;45:3139-52. 
[32] Ben Hamida A, Djeran-Maigre I, Dumontet H, Smaoui S. Clay compaction modelling by multiscale homogenization theory. Int J Rock Mech Mining 2005;42(7-8):996-1005.

[33] Smaoui S, Ben Hamida A, Djeran-Maigre I, Dumontet H. Micro-macro approaches coupled to an iterative process for nonlinear porous media. Comput Mater Continua 2006;4(3):153-62.

[34] Mounajed G, Boussa H, Grondin F, Menou A. Micro structural origin of the apparent thermal transient creep of concrete at high temperature. In: 11th International conference on fracture ICFXI; 2005.

[35] Bentz D, Garboczi E, Martys N. Application of digital-image-based models to microstructure, transport properties, and degradation of cement-based materials. In: Jennings $\mathrm{H}$, editor. Modelling of microstructure and its potential for studying transport properties and durability. Kluwer Academic Publisher; 1996. p. 167-85.

[36] Stroeven P, Stroeven M. Assessment of packing characteristics by computer simulation. Cem Concr Res 1999;29:1201-6.

[37] Guidoum A, Navi P, Huet C. 3d Numerical analysis of long term behaviour on concrete-like composite material. In: Proceedings of research and development in the field of dams, CIGB-ICOLD, Crans-Montana; 1995. p. 289-98.

[38] Huet C. Coupled size and boundary-condition effects in viscoelastic heterogeneous and composite bodies. Mech Mater 1999;31(12):787-829.

[39] Grassl P, Rempling R. A damage-plasticity interface approach to the mesoscale modelling of concrete subjected to cyclic compressive loading. Eng Fract Mech 2008;75:4804-18.

[40] Mounajed G. Conception et développement du modèle aléatoire béton numérique dans le code de calcul aux eléments finis symphonie, Research report cstb, CSTB, <http://mocad.cstb.fr>; 2001.

[41] Obeid W, Mounajed G, Alliche A. Experimental identification of biot's hydromechanical coupling coefficient for cement mortar. Mater Struct 2002;35:229-36.

[42] Ung Quoc H, Mounajed G. A new f.e. tangent damage model modev for concrete structures. In: 11th International conference on fracture ICFXI; 2005.

[43] Mazars J. Application de la mécanique de l'endommagement au comportement non linéaire et à la rupture du béton de structure, Thèse de doctorat, Université Pierre et Marie Curie; 1984.

[44] Aboudi J. Mechanics of Composite Materials: a unified micromechanical approach. Amsterdam: Elsevier Science Publishers B.V.; 1991.

[45] Chateau X, Dormieux L. Approche micromécanique du comportement d'un milieu poreux non saturé. CR Acad Sci Paris II B 1998;326:533-8.

[46] Dormieux L, Molinari A, Kondo D. Micromechanical approach to the behavior of poroelastic materials. J Mech Phys Solids 2002;50:2203-31.

[47] Dagan G. Flow and transport in porous formations. Springer-Verlag; 1989.

[48] Desbarats A. Numerical estimation of effective permeability in sand-sale formations. Water Resour Res 1987;23(2):273-86.

[49] Eberhard J, Attinger S, Wittum G. Coarse graining for upscaling of flow in heterogeneous porous media. Multiscale Model Simul 2004;2(2):269-301.

[50] Gomez-Hernandez J. A stochastic approach to the simulation of block conductivity fields conditioned upon data measured at a smaller scale. PhD thesis, Stanford University; 1991.

[51] Matheron G. Eléments pour une théorie des milieux poreux. Tech rep, Technip; 1967.

[52] Bear J, Bachmat Y. Introduction to modeling of transport phenomena. In: Theory and applications of transport in porous media. Kluwer Academic Publishers; 1991.

[53] Choinska M, Khelidj A, Chatzigeorgiou G, Pijaudier-Cabot G. Effects and interactions of temperature and stress-level related damage on permeability of concrete. Cem Concr Res 2007;37:7988.

[54] Goueygou M, Lafhaj Z, Kaczmarek M. Relationship between porosity, permeability and ultrasonic parameters in sound and damaged mortar. In: International symposium NDT-CE; 2003.

[55] Grondin F. Modélisation multi-échelles du comportement thermo-hydromécanique des matériaux hétérogènes - applications aux matériaux cimentaires sous sollicitations sévères, Phd thesis, Université Pierre et Marie Curie; 2005.

[56] Picandet V, Khelidj A, Bastian G. Effect of axial compressive damage on gas permeability of ordinary and high-performance concrete. Cem Concr Res 2001:31:1525-32.

[57] Bary B., Etude du couplage hydraulique-mécanique dans le béton endommagé. Thèse de doctorat, Ecole Normale Supérieure de Cachan; 1996.

[58] Grondin F, Bouasker M, Mounanga P, Khelidj A. Physico-chemical deformations of solidifying cementitious systems: multiscale modelling. Mater Struct 2010;43:151-65.

[59] Cui L, Cahyadi J. Permeability and pore structure of opc paste. Cem Concr Res 2001;31:277-82.

[60] Bentz D, Garboczi E, Haecker C, Jensen O. Effects of cement particle size distribution on performance properties of portland cement-based materials. Cem Concr Res 1999;29:1663-71.

[61] Constantinides G, Ulm F. The effect of two types of C-S-H on the elasticity of cement-based materials: results from nanoindentation and micromechanical modeling. Cem Concr Res 2003:2450.

[62] Chateau X, Dormieux L. Micromechanics of saturated and unsaturated porous media. Int J Numer Anal Methods Geomech 2002;26:831-44.

[63] Garboczi E, Bentz D. Modeling of the microstructure and transport properties of concrete. Construct Build Mater 1996;10(5):293-300.

[64] Katz A, Thompson A. Quantitative prediction of permeability in porous rock. Phys Rev B 1986;34(11):8179-81.

[65] Powers T. Structure and physical properties of hardened portland cement paste. J Am Ceram Soc 1958;41:1

[66] Haecker C-J, Garboczi E, Bullard J, Bohn R, Sun Z, Shah S, et al. Modeling the linear elastic properties of portland cement paste. Cem Concr Res 2005;35(10):1948-60.

[67] Ye G, Sun Z, Voigt T, van Breugel K, Shah S. A micromechanic model for characterization of cement paste at early age validated with experiments. In: RILEM editor. Int symp advances in concrete through science and engineering, Evanston, USA; 2004.

[68] Powers T, Copeland L, Hayes J, Mann M. Permeability of portland cement paste. J Am Concr Inst 1954;51:285.

[69] Khelidj A, Loukili A, Bastian G. Experimental study of the hydro-chemical coupling inside maturing concretes: effect on various types of shrinkage. Mater Struct 1998;31(213):588-94.

[70] Biot M. Theory of propagation of elastic waves in a fluid-saturated porous solid. J Acoust Soc Am 1956;28:168-91.

[71] Pimienta P, Hager I. Mechanical behaviour of hpc at high temperature. In: 6th International symposium on utilisation of high strength/high performance concrete; 2002

[72] Dreux G, Festa J. Nouveau guide du béton, Eyrolles; 1995.

[73] Tourenq C, Durville J-L, Massieu E., Les propriétés des roches: guide pratique. In: Mémento, Mines et Carrières, No. 3, LCPC; 1997.

[74] Malier Y. Les bétons à hautes performances: caractérisation, durabilité, applications, Presses de l'Ecole Nationale des Ponts et Chaussées; 1992.

[75] Kalifa P, Tsimbrovska M, Baroghel-Bouny V. High-performance concrete at elevated temperatures an extensive experimental investigation of thermal and hygral properties and microstructure. In: Proceedings of int symp on highperformance and reactive powder concretes, Sherbrooke, Canada; 1998. p. 259279.

[76] Tsimbrovska M, Kalifa P, Quenard D, Dayan J. High performance concrete at elevated temperature: Permeability and microstructure. In: Livolant M, editor. Transactions of the 14th international conference on structural mechanics in reactor technology, Lyon, France; 1997. p. 475-82.

[77] Rozière E, Loukili A, El Hachem R, Grondin F. Durability of concrete exposed to leaching and external sulphate attacks. Cem Concr Res 2009;39:1188-98.

[78] Pensée V, Dormieux L, Kondo D, Deudé V. Poroélasticité dun milieu mésofissuré : analyse micromécanique. CR Méc 2002;330:147152. 